\title{
TOP Detector for Particle Identification at the Belle II Experiment
}

\author{
Yasuyuki Horii* $\dagger$ \\ Nagoya University \\ E-mail: yhorii@hepl.phys.nagoya-u.ac.jp
}

The Belle II experiment is an upcoming experiment based on the SuperKEKB $e^{+} e^{-}$collider, which will provide almost two orders of magnitude higher instantaneous luminosity than the KEKB $e^{+} e^{-}$collider. We aim to measure precisely the parameters of the Standard Model and search for physics beyond the Standard Model. We report here on the time-of-propagation ("TOP") detector, which plays an essential role in the identification of charged particles at the Belle II experiment. Each TOP counter consists of a quartz radiator and 32 micro-channel-plate photomultiplier tubes ("MCP-PMTs"). The Cherenkov photons traveling in the quartz radiator are detected by the MCP-PMTs, and the time and the position are used to reconstruct the Cherenkov angle corresponding to the velocity. The key features of the TOP counter as well as a performance study using a full-size prototype are presented.

The European Physical Society Conference on High Energy Physics -EPS-HEP2013

18-24 July 2013

Stockholm, Sweden

\footnotetext{
* Speaker.

$\dagger$ On behalf of the Belle II PID Group.
} 


\section{Introduction}

The Belle II experiment $[1,2]$ is an upcoming experiment based on the SuperKEKB $e^{+} e^{-}$ collider, which will provide almost two orders of magnitude higher instantaneous luminosity than the KEKB $e^{+} e^{-}$collider. We aim to measure precisely the parameters of the Standard Model and search for physics beyond the Standard Model.

We report here on the time-of-propagation ("TOP”) detecter, which plays an essential role in the identification of charged particles at the Belle II experiment. Each TOP counter consists of a quartz radiator and 32 micro-channel-plate photomultiplier tubes ("MCP-PMTs"). The Cherenkov photons traveling in the quartz radiator are detected by the MCP-PMTs (Figure 1), and the time and the position are used to reconstruct the Cherenkov angle corresponding to the velocity. Combining with the momentum measured in the inner trackers, the mass is identified.

In total 16 TOP counters are planned to be installed in the barrel region of the Belle II detector. The expected efficiency of identifying a kaon or a pion is typically $95 \%$, while the expected probability ("fake rate") of misidentifying a pion (kaon) as a kaon (pion) is typically $5 \%$, both depending on the momentum. In the following, the key features of the TOP counter as well as a performance study using a full-size prototype are presented.

\section{Quartz Radiator}

A quartz radiator consists of two quartz bars, a quartz bar with mirror, and a wedge-shaped quartz block (Figure 2). The dimensions of a quartz bar, a quartz bar with mirror, and a wedgeshaped quartz block are $1250 \times 450 \times 20 \mathrm{~mm}^{3}, 100 \times 450 \times 20 \mathrm{~mm}^{3}$, and $100 \times 450 \times(20-50) \mathrm{mm}^{3}$, respectively, where the height of wedge-shaped block varies from $20 \mathrm{~mm}$ to $50 \mathrm{~mm}$.

The Cherenkov photons are emitted inside the quartz radiator, and are propagated with hundreds of reflections at the quartz surfaces and/or a reflection at the mirror. The quartz material is Corning 7980 Class 0 Grade F or better. The surfaces of the quartz radiator are polished with a roughness of $<0.5 \mathrm{~nm}$ in RMS to ensure photon efficiency. A flatness of $<6.3 \mu \mathrm{m}$, a perpendicularity of $<20$ arcsec, and a parallelism of $<4$ arcsec are required for the largest surfaces of the quartz bars to maintain the angles of the Cherenkov photons. The mirror has a curvature of a radius of $5000 \mathrm{~mm}$ to cope with chromatic dispersion. The wedge-shaped quartz block expands the Cherenkov photon path for a better photon separation.

We have established a system consists of laser and photodiodes to measure the bulk transmittance and the surface reflectance, which are the key parameters related to the photon efficiency. For a prototype bar, we obtain a bulk transmittance of $(99.4 \pm 0.2) \% / \mathrm{m}$ and a surface reflectance of more than $99.9 \%$ per bounce for various reflection angles in $55-70^{\circ}$. We have also established a system consists of laser and CCD to check the photon path inside the quartz. For a prototype bar, no significant change from the ideal path is observed. The systems for measuring the mirror reflectivity and the wedge angle have also been developed, and checks have been performed for prototypes.

The quartz components are glued together for forming a quartz radiator. The stage has been developed using solid aluminum plates, whose surface flatness is kept to be within $\pm 0.05 \mathrm{~mm}$ using micrometer heads. The quartz is put on hundreds of POM balls on the stage, where the quartz- 
surface flatness is kept to be within $\pm 0.01 \mathrm{~mm}$. The quartz components are aligned with a relative angle of $\mathscr{O}(0.01) \mathrm{mrad}$ and a displacement of $\mathscr{O}(0.01) \mathrm{mm}$. Norland Optical Adhesive 63, which is cured with ultra-violet light emission, is selected as the glue. We successfully glued prototype components and the first (almost-)full-size quartz radiator has been developed (Figure 3).

\section{Support Structure}

The quartz radiator is supported by hundreds of peek buttons attached on the aluminum honeycomb plates (Figure 4). The peek buttons are selected so that the quartz surfaces are not damaged. Since the refractive index of the peek buttons are different from air, the area of the contact between quartz and peek is kept small by using button shape. The aluminum honeycomb plates have an enough rigidity with low material volume. A prototype of the support structure has been developed, and the prototype quartz radiator has been installed.

\section{MCP-PMT}

The Cherenkov photons are detected by the MCP-PMTs (Figure 5). They have square shape for effectively covering the quartz-surface area. Single MCP-PMT has $4 \times 4$ channels in the active area of $23 \times 23 \mathrm{~mm}^{2}$. The Cherenkov photon is converted into an electron at the photocathode made of NaKSbCs. The quantum efficiency is $>24 \%$ at a wave length of $380 \mathrm{~nm}$. The produced electron is amplified by hitting the walls of the halls of two micro-channel plates ("MCPs"). The collection efficiency, which corresponds to the MCP aperture ratio, is 50-55\%. The gain is $2 \times 10^{6}$ at a bias voltage around $3.4 \mathrm{kV}$, which enables single photon detection. The transit time spread is $\sim 40 \mathrm{psec}$, which is essential for obtaining required particle-identification performance. The performance loss in a magnetic field of $1.5 \mathrm{~T}$, designed for the Belle II experiment, has been measured to be small. The mass production started in 2011, and the quality checks are ongoing.

\section{Readout}

The Cherenkov photon signal from the MCP-PMTs is read out using waveform-sampling ASICs [3, 4]. Currently-tested version of the ASIC is IRS3B. It has a high sampling rate of $4 \times 10^{9}$ samples per second. The chip intrinsic time resolution is $<25 \mathrm{psec}$. Multihit buffering is possible for $30 \mathrm{kHz}$ level-1 trigger accept, which is expected at the Belle II experiment. Four chips are attached on a carrier board, and four carrier boards form a module (Figure 6). A module corresponds to 16 MCP-PMTs. The readout is controlled by Xilinx Spartan-6 FPGA.

Since the calibration of the time and the charge for the above readout system requires a significant learning curve, an alternative system has been prepared as a backup for performance tests [5]. It is based on constant-fraction discriminators with CAEN V1290A TDCs. Four channels are converted into a single channel in the socket boards due to limited space for the electronics. Eight constant-fraction-discriminator boards form a module corresponding to two MCP-PMTs (Figure 7). The time resolution for this readout system is measured to be $\sim 50 \mathrm{psec}$. The expected performance loss of using this system relative to using the baseline system corresponds to a decrease of efficiency of $<1 \%$ at an increase of fake rate of $1 \%$. 


\section{Performance Study}

For evaluating the performance of the TOP counter, a test has been performed using a prototype counter and $2.0 \mathrm{GeV} / c$ positron beam at the Laser Electron Photon Beamline at SPring-8 ("LEPS"). Figure 8 represents the overview of the setup. The positron is produced at the lead target, and is bended by the magnetic field generated by the dipole magnet. The positrons with the momenta of $2.0 \mathrm{GeV} / c$ are tagged by the trigger counters, and the tracks are obtained by the scintillating-fiber trackers. The time origin is obtained from the radio-frequency pulse for the accelerator with a resolution of $<25 \mathrm{psec}$. The beam time is divided into two, one for the baseline readout and the other for the backup readout. The test has been done in a collaboration with the LEPS group.

Figure 9 shows a very preliminary output from the data taken with the backup readout and a GEANT 4-based Monte Carlo ("MC") expectation. The overall structure of the photon arrival time depending on the channels agree between the data and MC samples. The data analysis does not include full event selection, e.g. for removing shower background, while it includes a preliminary channel-by-channel time-origin alignment using the data taken with laser input. Further calibration and analysis are ongoing for evaluating the time resolution of photon arrival, the velocity resolution of tracks, and the performance of particle identification. The calibration and the analysis are also ongoing for the data taken with the baseline readout.

\section{Summary}

The TOP detecter will play an essential role in the charged particle identification at the Belle II experiment. The quartz radiator is designed to maintain Cherenkov-photon efficiency and paths. The MCP-PMTs enable the photon detection with a transit time spread of $\sim 40 \mathrm{psec}$ and a high quantum efficiency. Baseline readout with waveform sampling and an alternative readout with constant-fraction discriminator for performance test have been developed. Preliminary output from the data taken with $2.0 \mathrm{GeV} / c$ positron input at LEPS shows a good agreement with MC expectation. Further analysis of the beam data is ongoing for evaluating the performance of the charged particle identification. The mass production of each component is ongoing or to be started soon aiming for the installation in 2015.

\section{References}

[1] T. Abe et al., "Belle II Technical Design Report", arXiv:1011.0352, 2010.

[2] Z. Dolezal, "Belle II at SuperKEKB, a Super B Factory", PoS(EPS-HEP 2013)341.

[3] G. Varner, "Experience with the First Generation Deep Sampling ASICs IRS and BLAB3", Workshop on Timing Detectors: Electronics, Medical and Particle Physics Applications, Cracow, 2010.

[4] G. Varner, "Deeper Sampling CMOS Transient Waveform Recording ASICs", 2nd International Conference on Technology and Instrumentation in Particle Physics, Chicago, 2011.

[5] K. Inami, "TOP Counter Prototype R\&D”, 7th International Workshop on Ring Imaging Cherenkov Detectors, Cassis, 2010. 


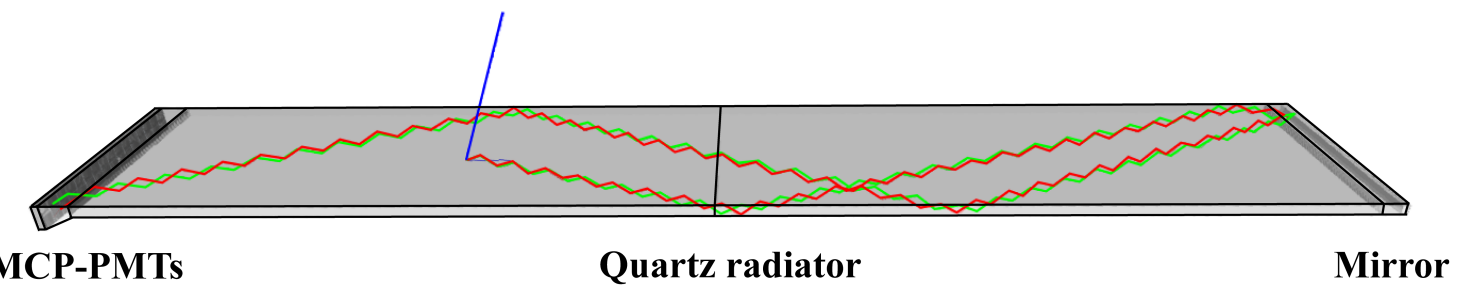

Figure 1: Schematic drawing of the TOP counter with examples of the Cherenkov-photon paths. The blue line represents the charged track, where a momentum of $2 \mathrm{GeV} / c$ is assumed. The green line indicates an example of the photon paths assuming charged-pion track, while the red line assuming charged-kaon track. The Cherenkov photons are detected at the MCP-PMTs after reflections at the quartz surfaces and the mirror. Tens of Cherenkov photons are typically detected for single track input.
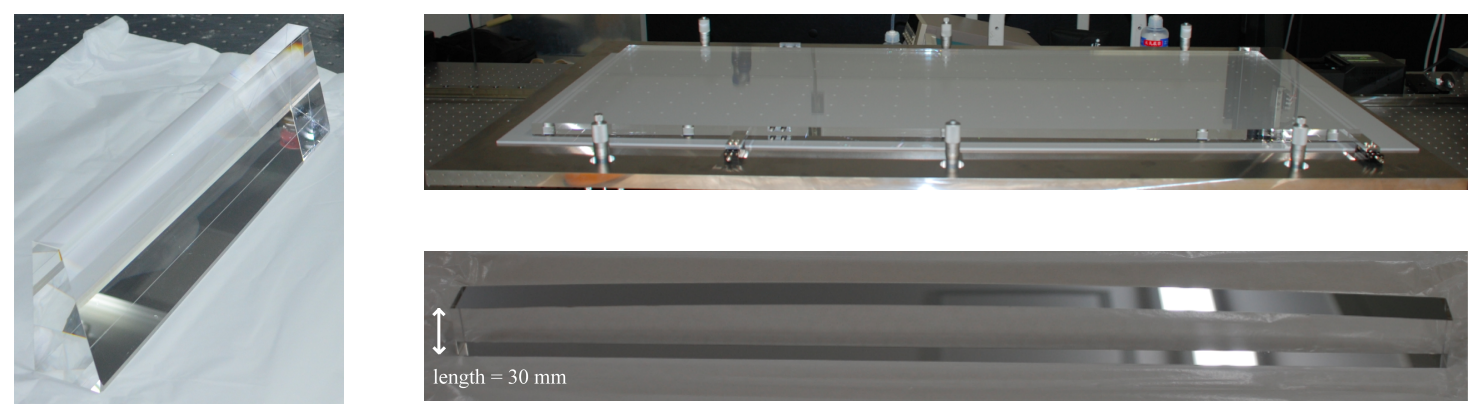

Figure 2: Prototypes of the quartz components. A wedge-shaped quartz block (left), a quartz bar (right top), and a quartz bar with mirror (right bottom) are shown. The length of the quartz with mirror is $30 \mathrm{~mm}$, which is $70 \mathrm{~mm}$ shorter than the design value. The material quality and the specifications related to polishing satisfy the design requirements.

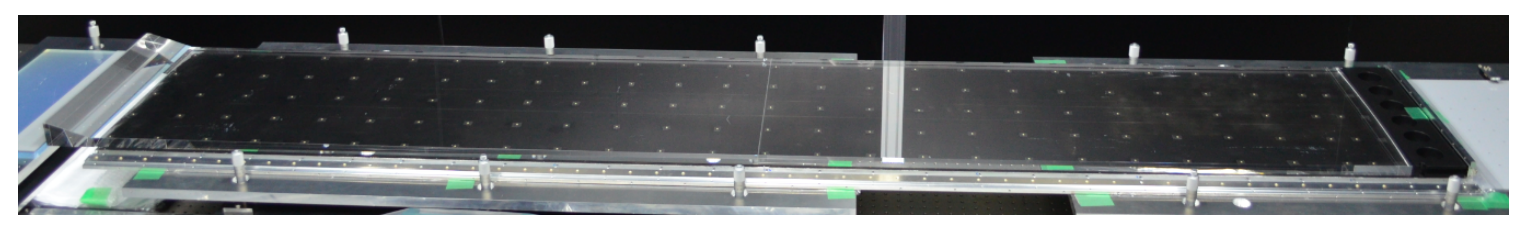

Figure 3: Prototype of the quartz radiator. A wedge-shaped quartz block, two quartz bars, and a quartz bar with mirror are glued. The radiator is put on peek buttons attached on an aluminum honeycomb plate. 

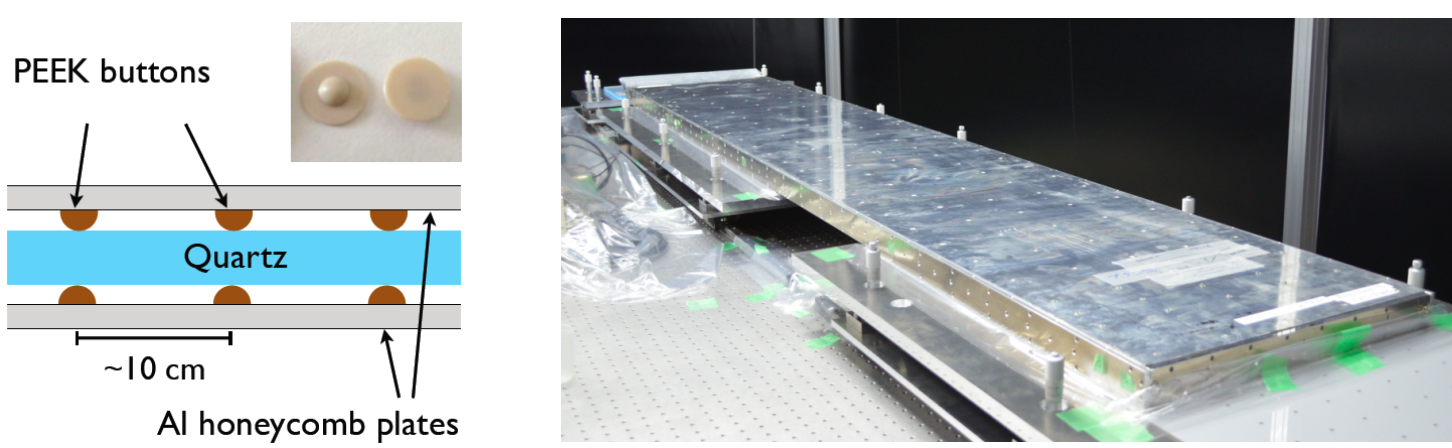

Figure 4: A schematic drawing of the support structure of the quartz radiator (left) and a picture of the support structure surrounding a quartz radiator (right). The quartz is supported by the peek buttons attached on the aluminum honeycomb plates.
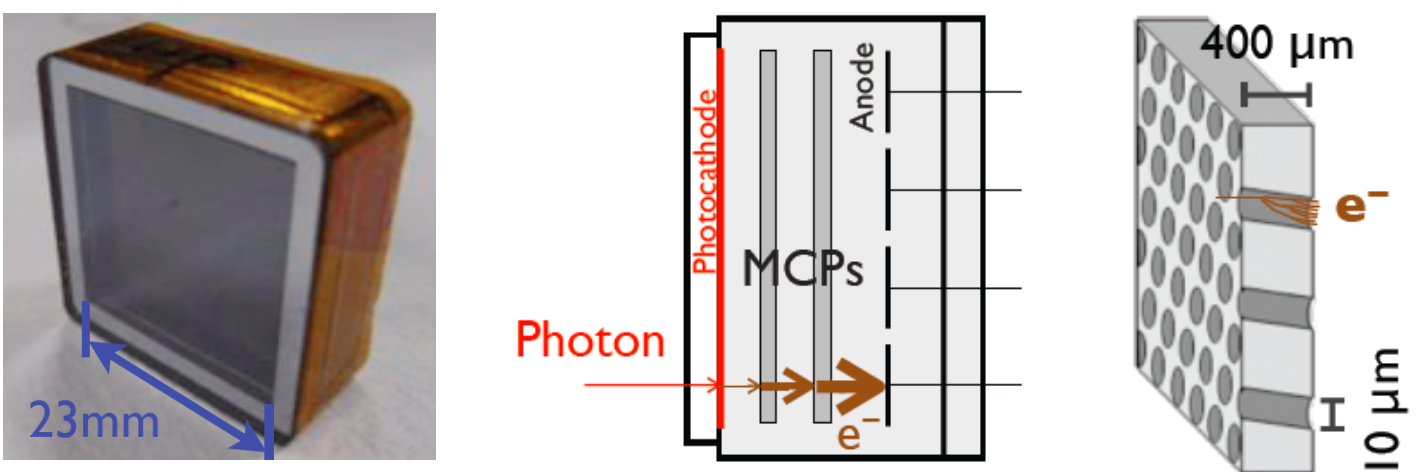

Figure 5: A picture of the MCP-PMT (left), a cross-sectional drawing of the MCP-PMT (center), and a schematic drawing of the MCP (right). Cherenkov photon is converted into electron, which is amplified by two MCPs.
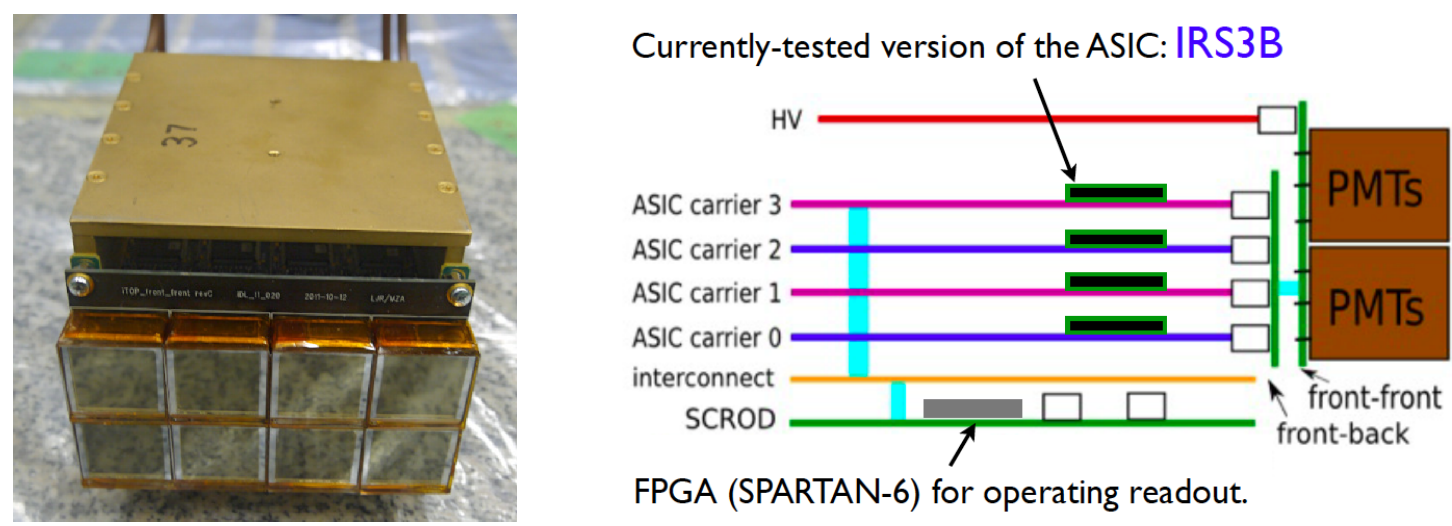

FPGA (SPARTAN-6) for operating readout.

Figure 6: A picture (left) and a schematic drawing (right) of the readout module with MCP-PMTs. 

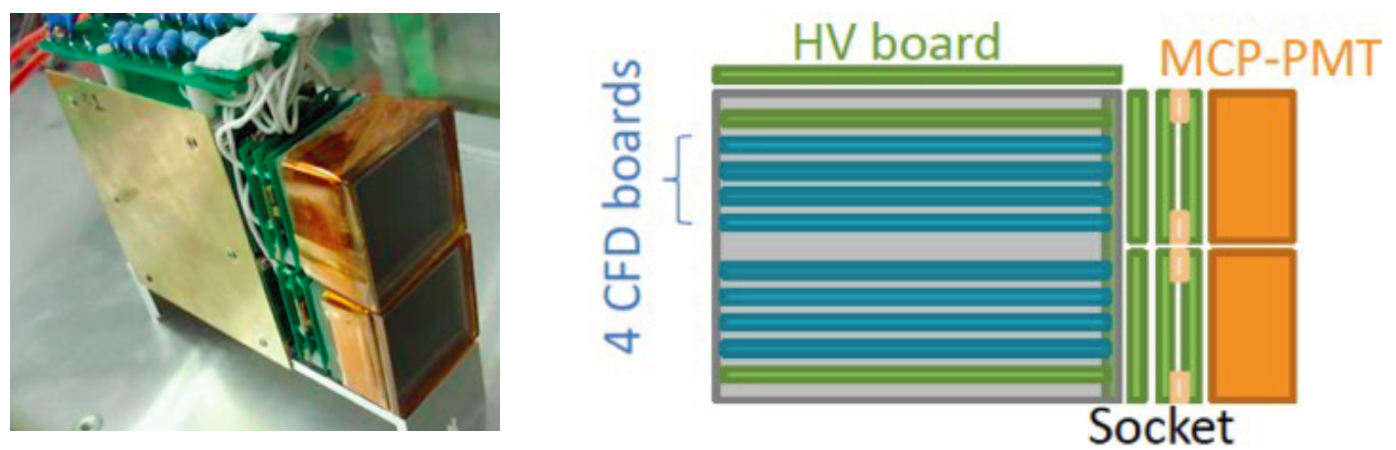

Figure 7: A picture (left) and a schematic drawing (right) of the backup readout module for performance test with MCP-PMTs.

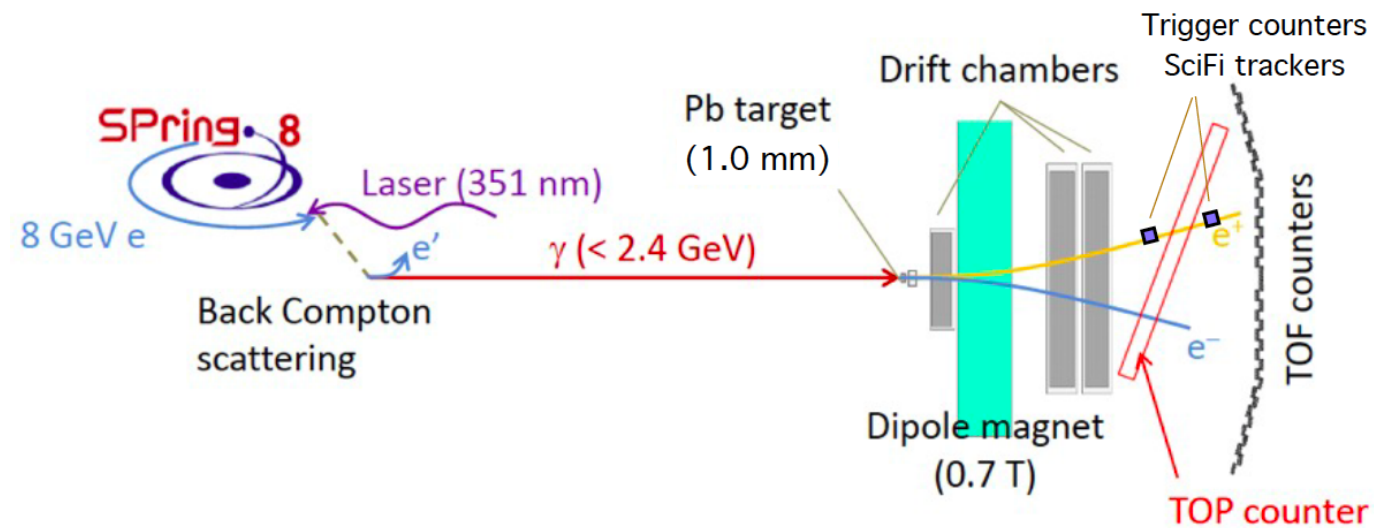

Figure 8: Setup of the performance test using $2.0 \mathrm{GeV} / c$ positrons at LEPS.
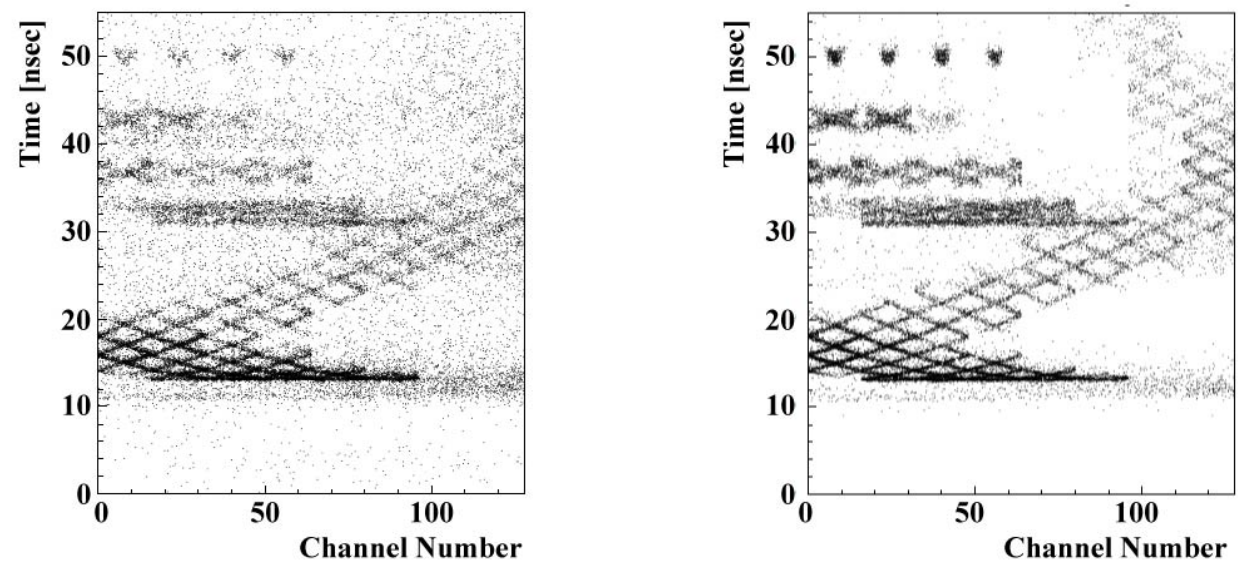

Figure 9: Preliminary ring images for data taken by the backup readout (left) and MC simulation (right). The horizontal axis shows the channel-identification number, while the vertical axis shows the time of photon detection at the corresponding channel. The events for normal incidence are accumulated. The two distributions have similar tendency. Further calibration and analysis are ongoing. 